\title{
Development of Efficient Packaging Method of Oligodeoxynucleotides by a Condensed Nano Particle in Lipid Envelope Structure
}

\author{
Yuma Yamada, ${ }^{a}$ Kentaro Kogure, $,{ }^{*}, b$ Yoshio Nakamura, ${ }^{a}$ Kaori Inoue, ${ }^{c}$ Hidetaka Akita,,${ }^{a, b}$ \\ Fumi Nagatsugi, ${ }^{d}$ Shigeki SAsaki, ${ }^{d}$ Tetsuya SuHARA ${ }^{c}$ and Hideyoshi Harashima ${ }^{a, b}$ \\ ${ }^{a}$ Graduate School of Pharmaceutical Sciences, Hokkaido University; Kita-12, Nishi-6, Kita-ku, Sapporo 060-0812, Japan: \\ ${ }^{b}$ CREST, Japan Science and Technology Agency (JST); 4-1-8 Honcho, Kawaguchi 332-0012, Japan: ${ }^{c}$ National Institute \\ of Radiological Sciences; 4-9-1 Anagawa, Inage-ku, Chiba 263-8555, Japan: and ${ }^{d}$ Graduate School of Pharmaceutical \\ Sciences, Kyushu University; 3-1-1 Maidashi, Higashi-ku, Fukuoka 812-8582, Japan.
}

Received April 12, 2005; accepted June 27, 2005; published online June 29, 2005

\begin{abstract}
An efficient delivery system is required if antisense oligodeoxynucleotides (ODN) are to be utilized for gene therapy. We report herein on the development of a novel ODN delivery system, ODN-encapsulated nano particles (ODN-ENP) using an efficient and simple packaging method. The ODN-ENP consists of a condensed ODN particle and a lipid envelope, which can be equipped with various functional devices for the efficient delivery of ODN with a small diameter $(150 \mathrm{~nm})$. The encapsulation efficiency and ODN recovery of ODN-ENP were significantly higher than those of other packaging methods, such as a stabilized antisense-lipid particles method or a freezethaw method. Furthermore, the time required for the preparation of the ODN-ENP was shorter than the other methods. The method developed in this study is a simple and efficient packaging method for ODN with a condensed nano particle in lipid-envelope structure.
\end{abstract}

Key words oligodeoxynucleotide; condensed particle; liposome; packaging; nano particle

Antisense synthetic oligodeoxynucleotides (ODN) are predicted to be a useful tool for gene therapy. ODN-mediated gene silencing has various advantages, such as technical simplicity, high stability and temporal effects. The antisense strategy using ODN has already been applied to the suppression of specific genes for growth of tumor and virus replication. ${ }^{1-9)}$ However, it is difficult to deliver the ODN to a target site, since it is susceptible to nucleases and penetrates the cell membrane with difficulty. ${ }^{10)}$ Semple et al. constructed a liposomal carrier for ODN, which they referred to as stabilized antisense-lipid particles (SALP) ${ }^{11)}$ for protection from nucleases and efficient cellular uptake. Their method is efficient for the encapsulation of ODN into liposomes by electrostatic interactions of negatively charged free ODN with $\mathrm{pH}$-sensitive cationic lipids. However, the SALP method is impractical, because the time required for preparation is lengthy and the encapsulation efficiency is low. Furthermore, SALP can not control the release of ODN from SALP in the cytoplasmic space.

We previously, proposed a novel packaging concept named a multifunctional envelope-type nano device (MEND) as an efficient and simple packaging method for plasmid DNA. ${ }^{12)}$ The MEND consists of a condensed core of plasmid DNA and a lipid envelope structure. The MEND has various advantages, since the lipid envelope structure can protect the condensed DNA from attack by nucleases and can be equipped with various functional devices such as polyethyleneglycole (PEG), to prolong blood circulation, a ligand for targeting and a $\mathrm{pH}$-sensitive fusogenic peptide to facilitate endosomal escape. ${ }^{13)}$ Furthermore, decondensation of the condensed DNA can be controlled by the condensing agents of the device. Therefore, this packaging concept would be expected to solve the problems described above, i.e., low encapsulation efficiency, lengthy preparation time and uncontrolled release in the cytosol. In the present study, we applied this concept to the packaging of ODN, and developed an efficient and simple delivery system for ODN by condensing
ODN and encapsulated it into nano particles.

\section{MATERIALS AND METHODS}

Materials Cholesterol (CH), 1,2-dioleoyl-3-dimethylammonium propane (DODAP), 1-palmitoyl-2-oleoyl-snglycerol-3-phosphocholine (POPC), didodecyldimethyl ammonium bromide (DDAB), and distearoyl phosphatidylethanolamine (DSPE)-polyethylene glycol (PEG) 2000 were purchased from AVANTI Polar Lipids Inc. (Alabaster, AL, U.S.A.). Cholesteryl hemisuccinate (CHEMS) was obtained from SIGMA-Aldrich Co. (St. Louis, MO, U.S.A.). Egg phosphatidylcholine (EPC), DSPE-PEG 2000-maleimide, and distearoyl phosphatidylcholine (DSPC) were purchased from the Nippon Oil and Fat Co. (Tokyo, Japan). Stearyl octaarginine (STR-R8) was synthesized ${ }^{14)}$ and generously provided by Dr. S. Futaki (Kyoto University, Japan).

Oligodeoxynucleotide (ODN) Synthesis The ODN for ACG 16 (5'-AGAAAGGAGAACAAAG-3'), and Pu-18 (5'AGGGAGGAGGGAGGAAGG-3') were synthesized on CPG supports using an Applied Biosystem 394 DNA/RNA synthesizer on a $1.0 \mathrm{mmol}$-scale using commercial 2-cyanoethyl phosphoamidites and the 6-FAM (purchased from Glen Research, Virginia, U.S.A.) according to the recommended protocol. The structure and purity of the ODN were confirmed by MALDI-TOF MS measurement. Fluorescent labeling of the ODN was conducted by conjugating fluorescein amide to $5^{\prime}$-terminal of the ODN.

Construction of ODN-ENP ODN-encapsulated nano particles (ODN-ENP) were constructed by the lipid film hydration method as reported previously. ${ }^{12)}$ The procedure consists of three steps as follows; 1) ODN (Pu-18) dissolved in $10 \mathrm{~mm}$ HEPES buffer (HB, $\mathrm{pH} 7.4)(0.36 \mathrm{mg} / \mathrm{ml})$ was mixed with an STR-R8 solution $(0.72 \mathrm{mg} / \mathrm{ml})$ to condense ODN under vortexing at a nitrogen/phosphate (N/P) ratio of 2.4 at room temperature. Then, $0.25 \mathrm{ml}$ of condensed ODN particles (COP) were added to the lipid film, formed by the 
evaporation of a chloroform solution of $625 \mathrm{nmol}$ lipids (EPC/CHEMS/DSPE-PEG2000/Rhodamine-DOPE $=8: 1$ : $1: 0.1$ (molar ratio)), on the bottom of a glass tube, followed by incubation for $10 \mathrm{~min}$ to hydrate the lipids. The glass tube was sonicated to produce ODN-ENP by coating the COP with lipids for about $15 \mathrm{~s}$ in a bath-type sonicator $(85 \mathrm{~W}$, Aiwa Co., Tokyo, Japan). ODN-ENP was then subjected to sucrose density gradient ultra-centrifugation. ${ }^{5)}$ A suspension of the ODN-ENP was layered on a discontinuous sucrose density gradient ( 0 to $60 \%$ ), and centrifuged at $160000 \times \boldsymbol{g}$ for $2 \mathrm{~h}$ at $20^{\circ} \mathrm{C}$. A $1 \mathrm{ml}$ aliquot was collected from the top and the fluorescence intensities of fluorescein and rhodamine were measured. The hydrodynamic diameter was measured by a quasi-elastic light scattering method and the zeta-potential was analyzed electrophoretically by means of an electrophoretic light scattering spectrophotometer (ELS-8000, Otsuka electronics, Japan).

Packaging of Free ODN by SALP Method and FreezeThaw Method

SALP Method Free ODN was packaged according to the preparation method of SALP. ${ }^{11)}$ The carrier was prepared using $13 \mu \mathrm{mol}$ of lipids (DSPC/CH/DODAP/DSPEPEG2000/DSPE-PEG2000-maleimide $=25: 45: 20: 8: 2$ (molar ratio)) and $200 \mu \mathrm{g}$ of ODN (ACG 16) containing the labeled ODN by an EtOH injection method. Sizing of carrier was achieved by extrusion. ${ }^{11)}$ Unencapuslated ODN were removed by size-exclusion chromatography.

Freeze-Thaw Method Free ODN was packaged according to encapsulation procedure of plasmid DNA with cationic lipids based on the freeze-thaw method. ${ }^{15)}$ The carrier was constructed using $20 \mu \mathrm{mol}$ lipids (POPC/ DDAB/DSPE-PEG 2000/DSPE-PEG 2000-maleimide $=94$ : $2: 3: 1$ (molar ratio)) and $100 \mu \mathrm{g}$ of ODN (ACG 16) by the freeze-thaw method. Sizing of carrier was achieved by extrusion. ${ }^{15)}$ The ODN absorbed to the surface of the liposomes and unencapsulated ODN were removed by size-exclusion chromatography after digestion with nuclease.
Calculation of Recovery Ratio of ODN and Lipid, and Encapsulation Efficiency The recovery of the lipids was determined by measuring phosphatidylcholine (PC) including EPC, DSPC, POPC with a PC C-test wako kit (Wako, Japan). ODN concentrations were determined by measuring the fluorescence intensity of the labeled ODN (excitation at $494 \mathrm{~nm}$ and emission at $518 \mathrm{~nm}$ ) after treatment with $1 \%$ TritonX-100. The recovery ratio of ODN and lipid were calculated as the recovered ODN and lipid divided by the applied ones, multiplied by 100 . Encapsulation efficiency was calculated as the final ODN to lipid ratio divided by the initial ODN to lipid ratio, multiplied by $100 .^{11)}$ Constructions of each carrier were operated two times respectively. Each value shown in Fig. 4 is represented by the mean $(n=2)$.

\section{RESULTS AND DISCUSSION}

Construction of ODN-ENP It is desirable to condense ODN with a polycation in order to enhance encapsulation efficiency and to control the intracellular release of ODN from the polycation. The condensation of ODN is an important step, because the size and charge of the condensed ODN particles (COP) are critical factors in the construction of ODNENP by the lipid hydration method (Fig. 1A). We therefore examined the effect of the ratio of polycation to ODN on hydrodynamic diameter and the zeta potential of COP and determined the conditions for ODN condensation for producing small and positively charged particles. We chose stearylated octaarginine (STR-R8) as a polycation, because STR-R8 is a good condenser of plasmid DNA. ${ }^{16)}$ As shown in Fig. 2, when the ODN was added to the STR-R8 solution, the diameter of the condensed particles was approximately $50 \mathrm{~nm}$ until the N/P ratio reach 1.6. Aggregation was observed at an $\mathrm{N} / \mathrm{P}$ ratio of between 1.0 and 1.6. The diameter was approximately $100 \mathrm{~nm}$ for $\mathrm{N} / \mathrm{P}$ ratios lower than 1.0 . In the region of $\mathrm{N} / \mathrm{P}$ above 1.6 , the zeta potential was positive $(20-7 \mathrm{mV})$, but became negative at N/P ratios below 1.0. This suggests
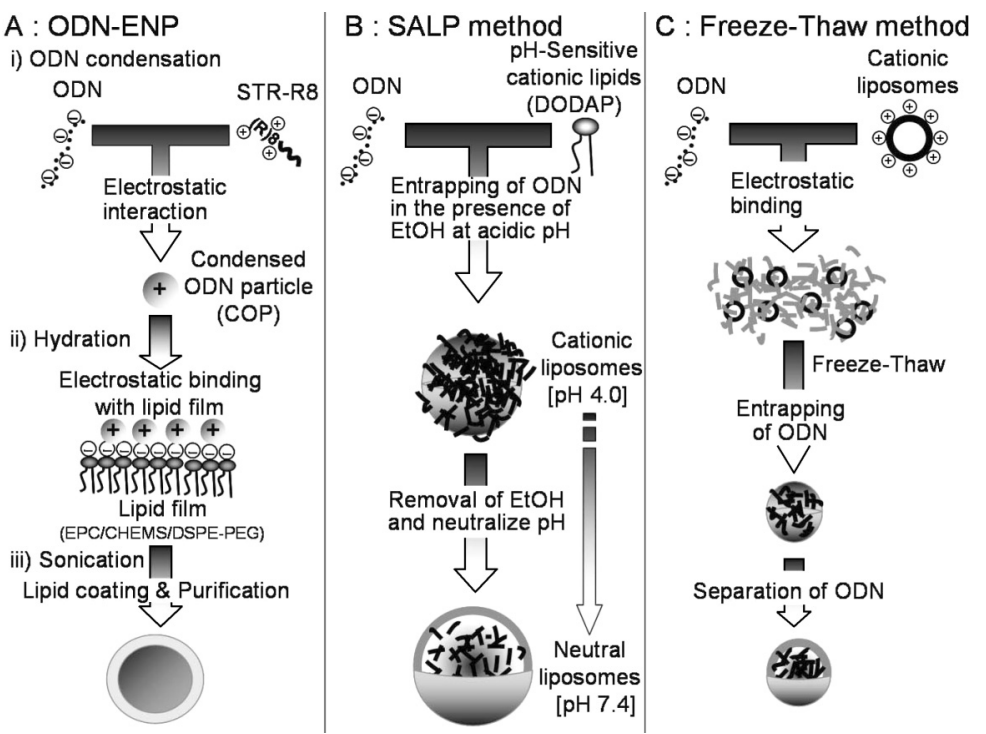

Fig. 1. Schematic Procedure of the Preparation of ODN-ENP, the SALP Method and Freeze-Thaw Method

(A) Procedure for preparing ODN-ENP: ODN was condensed electrostatically with STR-R8. Lipids were hydrated for binding to the condensed ODN particles (COP). The COP was coated with a lipid bilayer by sonication. (B) SALP method: free ODN was added to liposomes containing the pH-sensitive cationic lipid DODAP in the presence of $40 \%$ of ethanol at acidic $\mathrm{pH}$. The mixture was then dialyzed to remove ethanol and the $\mathrm{pH}$ adjusted to 7.4. (C) Freeze-Thaw method: free ODN was added to cationic liposomes, and the mixture subjected to freezing and thawing to encapsulate the ODN. 


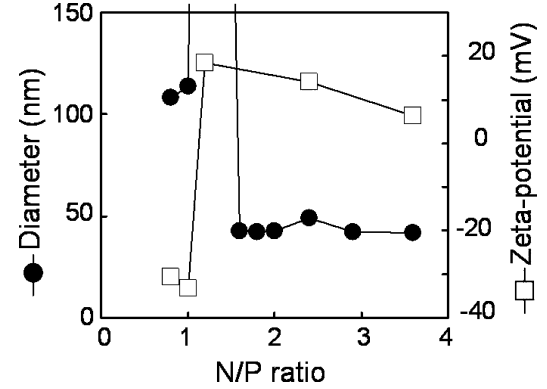

Fig. 2. Relationship between N/P Ratio and Hydrodynamic Diameter or Zeta Potential of Condensed ODN Particles

An ODN solution was added to an STR-R8 solution under vortexing at room temperature and the hydrodynamic diameter and zeta potential were measured. Closed circles; hydrodynamic diameter, open squares; zeta potential.

Table 1. Characteristics of ODN-ENP and Carriers Prepared by SALP Method and Freeze-Thaw Method

\begin{tabular}{lrrc}
\hline \hline & $\begin{array}{c}\text { Diameter } \\
(\mathrm{nm})\end{array}$ & $\begin{array}{c}\text { Zeta potential } \\
(\mathrm{mV})\end{array}$ & $\begin{array}{c}\text { Time required } \\
\text { preparation }(\mathrm{h})\end{array}$ \\
\hline COP & $73.8 \pm 4.4$ & $18.1 \pm 2.2$ & - \\
ODN-ENP & $150.0 \pm 9.8$ & $-4.3 \pm 2.3$ & 4 \\
SALP method & $188.3 \pm 2.3$ & $-1.3 \pm 0.5$ & 16 \\
Freeze-Thaw method & $95.6 \pm 3.2$ & $1.7 \pm 0.6$ & 7 \\
\hline
\end{tabular}

that aggregation was caused by the elestrostatic neutralization of ODN and STR-R8. Based on these results, condensed ODN particles (COP), which are small and positively charged, were prepared at an N/P ratio of 2.4. A suspension of COP was next added to the lipid film containing the negatively charged lipid CHEMS, followed by incubation to hydrate the lipid and to induce electrostatic binding. The COP was then packaged in a lipid bilayer by sonication. The lipidcoated particle is referred to as an ODN-encapsulated nano particle (ODN-ENP). The diameter of the ODN-ENP was larger than COP, and the zeta potential of the COP became opposite as the result of the packaging (Table 1). As shown in Fig. 3, after the ODN-ENP was purified by sucrose density gradient centrifugation of the sonicated sample, ODN and lipid were observed in the same fraction (\# 9). Furthermore, solubilization of the fraction increased the fluorescence intensity of fluorescein by about 12-fold (data not shown), clearly indicative of fluorescence resonance energy transfer between fluorescein-labeled ODN and the rhodamine-labeled lipid. This indicates that the positively charged COP was coated with a negatively charged lipid bilayer.

Comparison of ODN-ENP with Other Carriers We compared the efficiencies of ODN encapsulation, ODN recovery and lipid recovery among ODN-ENP and other, previously reported packaging methods. ${ }^{11,15)}$ In the SALP method, free ODN were encapsulated into liposomes by electrostatic interaction with the $\mathrm{pH}$-sensitive cationic lipid DODAP in the presence of $40 \%$ of ethanol at acidic condition ( $\mathrm{pH} 4.0$ ) (Fig. 1B). The special feature of this method is the removal of free ODN bound to the surface of liposomes by reducing the cationic charge at neutral $\mathrm{pH}$. The diameter of the carrier prepared by the SALP method was $188 \mathrm{~nm}$, slightly larger than that for the ODN-ENP.

On the other hand, the Freeze-Thaw method is a conventional encapsulating method for free plasmid DNA ${ }^{15}$ ) (Fig.

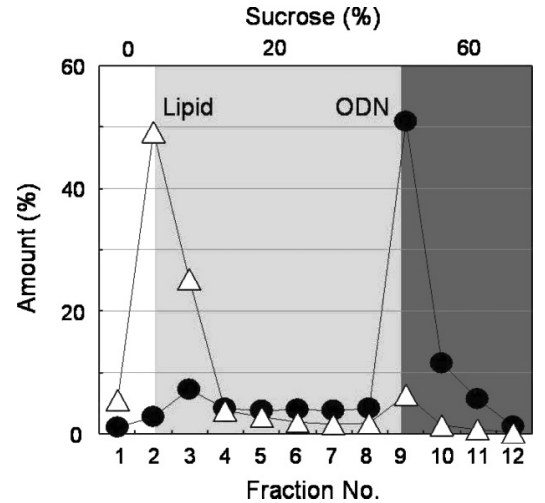

Fig. 3. Discontinuous Sucrose Density Gradient Ultra Centrifugation of ODN-ENP

The amounts of ODN (closed circle) and lipids (open triangle) were determined by the fluorescence intensities of fluorescein-labeled ODN and rhodamine-labeled lipid, respectively, after the solubilization of ODN-ENP by $0.1 \%$ SDS.

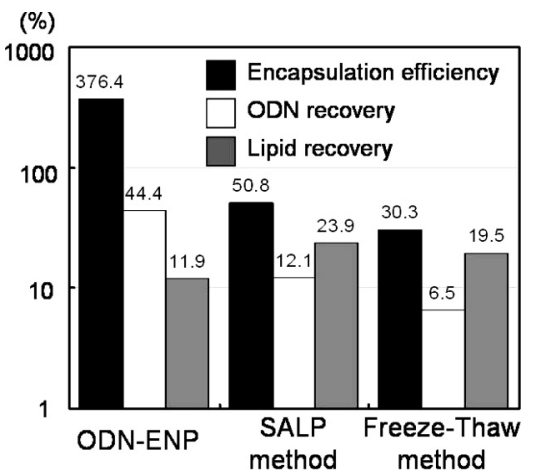

Fig. 4. Comparison of Encapsulation Efficiency, Recovery of ODN, and Recovery of Lipid

Encapsulation efficiencies were calculated as the ratio of the final ODN to lipid ratio devided by the initial ODN to lipid ratio, multiplied by 100 . The percentage recovery of ODN and lipid were calculated as the recovered ODN and lipid divided by the applied ones, multiplied by 100 . Data are represented by the mean $(n=2)$

1C). In the Freeze-Thaw method, free ODN is encapsulated by the freezing and thawing of the mixture of free ODN and cationic liposomes. The prepared carrier had a very small diameter $(96 \mathrm{~nm})$ and a slight positive charge (Table 1).

The calculated encapsulation efficiency of ODN-ENP was $380 \%$, due to the fact that ODN-ENP significantly enhances the encapsulation efficiency of ODN by lipid by forming condensed ODN particles. The encapsulation efficiency of ODN-ENP was much higher than those for other methods such as the SALP method and the Freeze-Thaw method (Fig. 4). This indicates that ODN were concentrated by about 4 times in ODN-ENP. Furthermore, the ODN-ENP showed the highest percentage recovery of ODN (44\%) of the three carriers (Fig. 4). However, the lipid recovery percentage values were similar. In addition, the time required for the preparation of ODN-ENP was significantly less than for both the SALP and Freeze-Thaw methods (Table 1). This, therefore, suggests that the method described here is the most efficient for the encapsulation of ODN into liposomes among the three methods. The condensation of ODN by polycation is responsible for the high packaging efficiencies of ODN, because the condensation of ODN was almost $100 \%$ and the lipid coating involved electrostatic interactions between the lipid bilayer and the condensed particle surface. Moreover, 
the condensed core structure has further advantages, i.e., the condensed ODN can be released into the cytosol, a process that can be controlled by optimizing the decondensation of ODN with optimized polycations.

In conclusion, we report on the successful development of an efficient and simple method for packaging ODN as the ODN-ENP. The ODN-ENP showed significantly higher efficiencies of encapsulation and recoveries of ODN than other encapsulating methods for free ODN. Therefore, the ODNENP are capable of serving as useful carriers for the efficient delivery of ODN by introducing various functional devices. In the future, we would introduce ODN-ENP to Tf and GALA system, ${ }^{17,18)}$ which delivers an encapsulated drug to cytosol efficiency, to construct practical application for cytoplasmic ODN delivery.

Acknowledgments We thank Dr. S. Futaki for providing STR-R8. This work was supported, in part, by Grants-in-Aid for Scientific Research (B) from the Ministry of Education, Culture, Sports, Science and Technology of Japan, and by Grants-in-Aid for Scientific Research on Priority Areas from the Japan Society for the Promotion of Science.

\section{REFERENCES}

1) Park W. S., Miyano-Kurosaki N., Abe T., Takai K., Yamamoto N., Takaku H., Biochem. Biophys. Res. Commun., 270, 953-960 (2000).

2) Stuart D. D., Kao G. Y., Allen T. M., Cancer Gene Ther., 7, 466-475 (2000).

3) Robaczewska M., Guerret S., Remy J. S., Chemin I., Offensperger W.
B., Chevallier M., Behr J. P., Podhajska A. J., Blum H. E., Trepo C., Cova L., Gene Ther, 8, 874-881 (2001).

4) Brignole C., Pagnan G., Marimpietri D., Cosimo E., Allen T.M., Ponzoni M., Pastorino F., Cancer Lett., 197, 231-235 (2003).

5) Matsuno A., Nagashima T., Med. Electron. Microsc., 37, 158-161 (2004).

6) Yuan J., Cheung P. K., Zhang H., Chau D., Yanagawa B., Cheung C., Luo H., Wang Y., Suarez A., McManus B. M., Yang D., Lab. Invest., 84, 703-714 (2004).

7) Wang M., Tsou T. H., Chen L. S., Ou W. C., Chen P. L., Chang C. F., Fung C. Y., Chang D., Hum. Gene Ther., 15, 1077-1090 (2004).

8) Pastorino F., Brignole C., Marimpietri D., Di Paolo D., Zancolli M., Pagnan G., Ponzoni M., Ann. N.Y. Acad. Sci., 1028, 90-103 (2004).

9) Yamanaka K., Gleave M.E., Hara I., Muramaki M., Miyake H., Mol. Cancer Ther., 4, 187-195 (2005).

10) Shi F., Hoekstra D., J. Control. Release, 97, 189-209(2004).

11) Semple S. C., Klimuk S. K., Harasym T. O., Dos Santos N., Ansell S. M., Wong K. F., Maurer N., Stark H., Cullis P. R., Hope M. J., Scherrer P., Biochim. Biophys. Acta, 1510, 152-166 (2001).

12) Kogure K., Moriguchi R., Sasaki K., Ueno M., Futaki S., Harashima H., J. Control. Release, 98, 317-323 (2004).

13) Kamiya H., Akita H., Harashima H., Drug Discov. Today, 8, 990—996 (2003).

14) Futaki S., Ohashi W., Suzuki T., Niwa M., Tanaka S., Ueda K., Harashima H., Sugiura Y., Bioconjug. Chem., 12, 1005-1011 (2001).

15) Shi N., Pardridge W. M., Proc. Natl. Acad. Sci. U.S.A., 97, 75677572 (2000).

16) Khalil I. A., Futaki S., Niwa M., Baba Y., Kaji N., Kamiya H., Harashima H., Gene Ther., 11, 636-644 (2004).

17) Kakudo T., Chaki S., Futaki S., Nakase I., Akaji K., Kawakami T., Maruyama K., Kamiya H., Harashima H., Biochemistry, 43, 56185628 (2004).

18) Yamada Y., Shinohara Y., Kakudo T., Chaki S., Futaki S., Kamiya H., Harashima H., Int. J. Pharmaceut. (in press). 